\title{
Discovery of a low-luminosity spiral DRAGN ${ }^{\star}$
}

\author{
D. D. Mulcahy ${ }^{1}$, M. Y. Mao ${ }^{1}$, I. Mitsuishi ${ }^{2}$, A. M. M. Scaife ${ }^{1}$, A. O. Clarke ${ }^{1}$, Y. Babazaki ${ }^{2}$, H. Kobayashi ${ }^{2}$, \\ R. Suganuma ${ }^{2}$, H. Matsumoto ${ }^{3}$, and Y. Tawara ${ }^{2}$ \\ 1 Jodrell Bank Centre for Astrophysics, Alan Turing Building, School of Physics and Astronomy, The University of Manchester, \\ Oxford Road, Manchester, M13 9PL, UK \\ e-mail: david.mulcahy@manchester.ac.uk \\ 2 Division of Particle and Astrophysical Science, Nagoya University, Furo-cho, Chikusa-ku, Nagoya, Aichi 464-8602, Japan \\ 3 Kobayashi-Maskawa Institute for the Origin of Particles and the Universe, Nagoya University, Furo-cho, Chikusa-ku, \\ Nagoya, Aichi 464-8602, Japan
}

Received 16 August 2016 / Accepted 13 September 2016

\begin{abstract}
Standard galaxy formation models predict that large-scale double-lobed radio sources, known as DRAGNs, will always be hosted by elliptical galaxies. In spite of this, in recent years a small number of spiral galaxies have also been found to host such sources. These so-called spiral DRAGNs are still extremely rare, with only $\sim 5$ cases being widely accepted. Here we report on the serendipitous discovery of a new spiral DRAGN in data from the Giant Metrewave Radio Telescope (GMRT) at $322 \mathrm{MHz}$. The host galaxy, MCG+07-47-10, is a face-on late-type Sbc galaxy with distinctive spiral arms and prominent bulge suggesting a high black hole mass. Using WISE infra-red and GALEX UV data we show that this galaxy has a star formation rate of $0.16-0.75 M_{\odot} \mathrm{yr}^{-1}$, and that the radio luminosity is dominated by star-formation. We demonstrate that this spiral DRAGN has similar environmental properties to others of this class, but has a comparatively low radio luminosity of $L_{1.4 \mathrm{GHz}}=1.12 \times 10^{22} \mathrm{~W} \mathrm{~Hz}^{-1}$, two orders of magnitude smaller than other known spiral DRAGNs. We suggest that this may indicate the existence of a previously unknown low-luminosity population of spiral DRAGNS.
\end{abstract}

Key words. galaxies: spiral - galaxies: jets - radio continuum: galaxies

\section{Introduction}

Spiral Double-lobed Radio sources Associated with Galactic Nuclei (DRAGNs, Leahy 1993) are spiral galaxies that host large-scale double-lobed radio sources. The existence of such sources contradicts our existing models of galaxy formation (e.g. Hopkins et al. 2008), which predict that DRAGNs should be hosted exclusively by elliptical galaxies. Until recently, observations of DRAGNs in the local Universe confirmed this expectation (e.g. Matthews et al. 1964; Urry \& Padovani 1995; Best et al. 2005).

Elliptical galaxies are formed as a result of mergers, the phenomenology of which also triggers the formation of DRAGNs (Chiaberge \& Marconi 2011; Chiaberge et al. 2015). However, a spiral galaxy's structure cannot withstand a major merger. Moreover, morphological transition from spiral to elliptical is thought to be a one-way process, at least in the local Universe. Consequently, the standard galaxy formation model does not predict the existence of spiral DRAGNs.

Nonetheless, a number of spiral DRAGNs have been discovered in recent years (e.g. Ledlow et al. 2001; Hota et al. 2011; Bagchi et al. 2014; Mao et al. 2015; Singh et al. 2015). While the first three spiral DRAGN discoveries were serendipitous, Mao et al. (2015) performed the first systematic search for these sources. Using the crowdsourced project Galaxy Zoo

\footnotetext{
* FITS cutout image of the observed spiral DRAGN MCG+07-4710 is only available at the CDS via anonymous ftp to cdsarc.u-strasbg. fr (130.79.128.5) or via http://cdsarc.u-strasbg.fr/viz-bin/qcat?J/A+A/595/L8
}

(Lintott et al. 2008), the authors cross-matched morphological classifications with the Faint Images of the Radio Sky at TwentyCentimeters (FIRST, Becker et al. 1995) and the NRAO VLA Sky-Survey (NVSS, Condon et al. 1998). In this study, only one spiral DRAGN was found above $L_{1.4 \mathrm{GHz}}=10^{23} \mathrm{~W} \mathrm{~Hz}^{-1}$. Singh et al. (2015) performed a similar analysis using the spiral galaxy catalogue of Meert et al. (2015) and reported the identification of four spiral DRAGNs in these data, including one that was previously known and three that were unknown. However, the precise identification of spiral DRAGN hosts remains contentious in the literature, and to date, only five spiral DRAGNs are widely accepted.

DRAGNs with spiral hosts may represent a rare phenomenon of elliptical galaxies transitioning back into spirals through accretion of gas and stars, perhaps from a companion. A key question is whether spiral DRAGNs are a result of non-standard physical properties, a result of their environment, or perhaps a combination of their nature and nurture. Studying spiral DRAGNs, as well as establishing their numbers more exactly, is vital to reconciling their role in standard galaxy formation theories.

In this Letter, we present the discovery of a new spiral DRAGN at $325 \mathrm{MHz}$ with the Giant Meterwave Radio Telescope (GMRT; Swarup 1990). In Sect. 2 we outline the data processing and imaging steps. In Sect. 3 we present the discovery of this new spiral DRAGN with a description of its radio morphology and that of the host galaxy, as understood from available multi-wavelength data. In Sect. 4, using infra-red and UV data, we demonstrate that the host galaxy is star-forming 
and we compare its star formation rate to other spiral DRAGNs. Finally, in Sect. 5 we discuss the nature of this object and state our conclusions. In this work we assume a $\Lambda$ CDM cosmology with $H_{0}=69.6 \mathrm{~km} \mathrm{~s}^{-1} \mathrm{Mpc}^{-1}, \Omega_{\mathrm{m}}=0.286$, and $\Omega_{\Lambda}=0.714$ (Bennett et al. 2014), which we use to calculate distance, luminosity and star formation rate. At a redshift of $z=0.017$, these values result in a conversion of $0.348 \mathrm{kpc} /{ }^{\prime \prime}$. All uncertainties are quoted at $1 \sigma$.

\section{Observations and data reduction}

Observations of the galaxy groups NGC 7618 and UGC 12491 were performed in full synthesis mode with the GMRT at $325 \mathrm{MHz}$. The GMRT is a full aperture synthesis telescope located near Pune, India (Swarup 1990). It consists of 30 steerable dishes that are $45 \mathrm{~m}$ in diameter, with a longest interferometric baseline of $25.5 \mathrm{~km}$, and 14 additional antennas located in a central $1 \mathrm{sq} . \mathrm{km}$, to provide dense $u v$ coverage at short spacings. The telescope operates at six frequencies: 150, 230, 325, 610, and $1420 \mathrm{MHz}$. In this work the $325 \mathrm{MHz}$ receiver was used. The full width at half maximum (FWHM) of the GMRT primary beam at $325 \mathrm{MHz}$ is approximately $81^{\prime} \pm 4^{\prime}$.

The observation took place on 16 August 2014 under project code 25.059 (PI Mitsuishi). The observation was a single pointing with a phase centre of $(\mathrm{J} 2000) 23^{\mathrm{h}} 19^{\mathrm{m}} 19^{\mathrm{s}}+42^{\circ} 54^{\prime} 50^{\prime \prime}$. The bright radio source $3 \mathrm{C} 48$ was used as a flux calibrator, tied to the flux density scale of Scaife \& Heald (2012).

The dataset was reduced using the Source Peeling and Atmospheric Modelling (SPAM) software (Intema et al. 2009). The SPAM software performed an initial phase calibration and astrometry correction using a sky model derived from the NVSS (Condon et al. 1998), followed by three rounds of directionindependent phase-only self-calibration. Following this, SPAM performed facet-based direction-dependent calibration on strong sources within the primary beam FWHM, and used the solutions from this calibration to fit a global ionospheric model. During this calibration process, various automated flagging routines were used between cycles of imaging and self-calibration to reduce residual RFI and clip statistical outliers.

The final primary-beam-corrected image towards MCG+0747-10 is shown in Fig. 1 and has a resolution of $9.4 \times 8.3^{\prime \prime}$. Images were made using an AIPS ROBUST value of -1.0. This parameter value was selected to achieve the best compromise between sensitivity and resolution. More details of these observations and data reduction can be found in Mitsuishi et al. (in prep.).

To isolate the integrated flux density of the separate source components (radio lobes and core), the source finding software, the Python Blob Detection and Source Measurement software, $\left(\mathrm{PYBDSM}^{1}\right)$ we initially used the source finding software, the Python Blob Detection and Source Measurement software to identify confusing compact sources. To do this, a local rms noise value $(\sigma)$ was calculated to identify all significant peaks above a threshold of $7 \sigma$. Following standard practice, PYBDSM then formed islands of contiguous emission down to a threshold of $5 \sigma$, and an elliptical Gaussian was fitted to each island, of which 625 were found in the entire field. Each Gaussian was then subtracted from the image, and the residual data were used to determine the integrated flux of the radio lobes. The measured integrated fluxes are listed in Table 1. The figures shown in this Letter are not the source-subtracted images.

\footnotetext{
1 https://dl.dropboxusercontent.com/u/1948170/html/ index.html
}

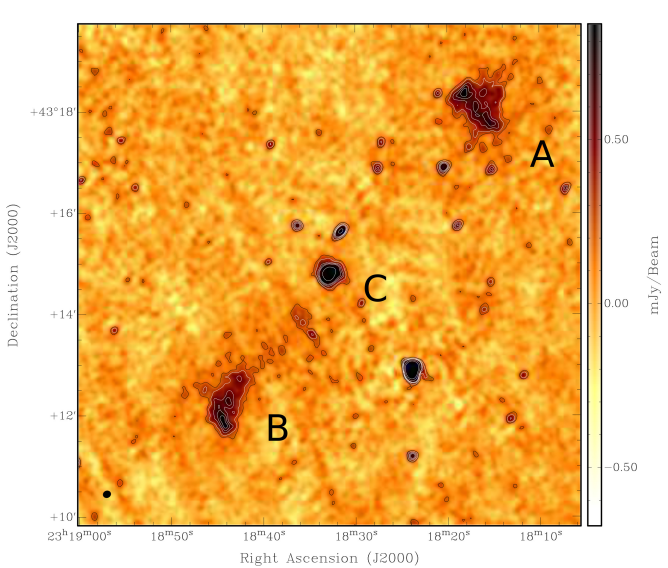

Fig. 1. Spiral DRAGN observed at $322 \mathrm{MHz}$. The local rms noise around the source is approximately $\sigma_{\mathrm{rms}}=68 \mu \mathrm{Jy} / \mathrm{beam}$. Contours are shown at $3,5,8,10,12,24$ and $48 \sigma_{\text {rms }}$. The GMRT synthesized beam has dimensions of $9.4 \times 8.3^{\prime \prime}$ and is shown in the bottom left corner as a filled-in ellipse.

\section{Discovery of spiral DRAGN MCG+07-47-10}

At a distance of approximately $22^{\prime}$ from the field phase centre, a Fanaroff-Riley type II radio galaxy is identified. This radio galaxy is shown in Fig. 1, where the lobes of this object are denoted A and B and the central host galaxy, MCG+07-47-10, is C. Closeups of the individual radio lobes are shown in Fig. 2. Coordinates for each of the lobes and the host galaxy are listed in Table 1. In particular, lobe B displays weak extended emission tracing back to the host galaxy (see the right panel in Fig. 2). Such weak emission is only now detectable through the high sensitivity of this observation and would have not been detected in surveys such as the NVSS or FIRST.

On inspection of the Digitized Sky Survey (DSS), the host galaxy (denoted C in Fig. 1), MCG+07-47-10, is a late-type galaxy with a classification of Sbc (Vorontsov-Vel'Yaminov \& Arkhipova 1968), a galaxy with spiral arms and a bulge. The limited resolution of the DSS means that the presence of a bar cannot be ruled out. The optical DSS image of this galaxy is shown in Fig. 3 with the $5 \sigma$ radio contour overlaid. The spiral arms of this face-on galaxy are clearly visible, and extended radio emission is seen coincident with the optical disk. If this emission were solely due to the core of the AGN, one would expect highly compact emission, unresolved by the GMRT in these data. Figure 1 shows that the radio emission in this case is clearly resolved, with a diameter measured from the $3 \sigma$ contour of approximately $45^{\prime \prime}$, equivalent to five synthesized beam widths. Such extended radio emission indicates that active star formation is occurring throughout the disk and is due to the injection of cosmic ray electrons (CREs) into the interstellar medium (ISM) by the action of supernovae. These CREs produce non-thermal synchrotron emission, which is observable here.

This area is not covered by the Sloan Digital Sky Survey (SDSS ${ }^{2}$; Alam et al. 2015), and no spectroscopic redshift information is currently available for this galaxy. However, MCG+07-47-10 is located close to the galaxy groups NGC 7618 $(z=0.0173)$ and UGC $12491(z=0.0173)$, the central galaxies of two nearby, galaxy groups of approximately equal mass. Kraft et al. (2006) found through X-ray observations that these galaxy groups are in the process of merging. Assuming that MCG+07-47-10 is associated with one of these galaxy groups, we adopt an approximate redshift of $z \approx 0.017$. At this

\footnotetext{
2 http://www.sdss.org/
} 

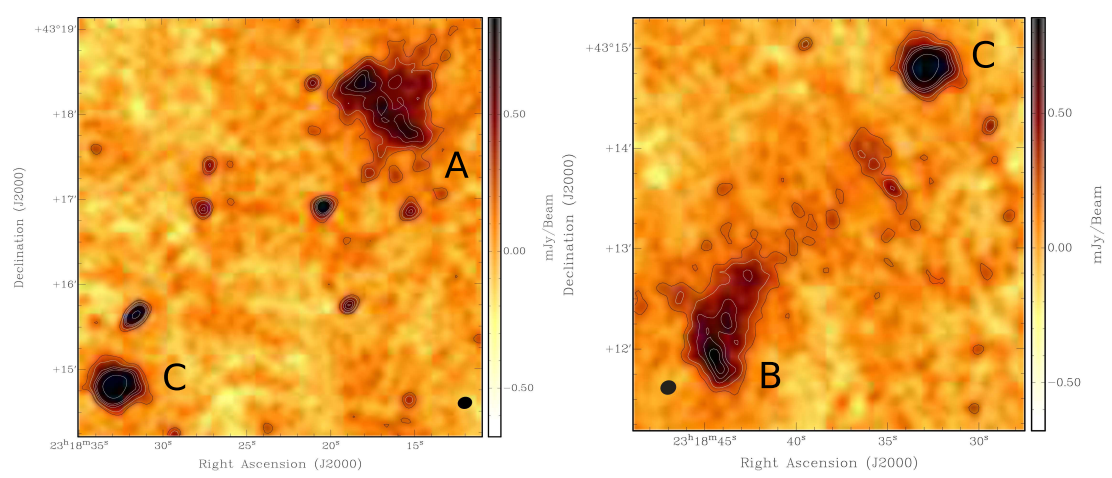

Fig. 2. Left: zoomed-in image of the northern lobe and host galaxy from Fig. 1. Right: zoomed-in image of the southern lobe and host galaxy from Fig. 1 . The measured rms noise $(\sigma)$ around the source is approximately $68 \mu \mathrm{Jy} /$ beam. The contours for both images are at levels of $3,5,8,10,12$, 24 and $48 \times \sigma$. The resolution is $9.4 \times 8.3^{\prime \prime}$ and is shown in the bottom right and left corner as a filled-in ellipse.

Table 1. Locations of the radio lobes and host galaxy of the spiral DRAGN with integrated fluxes and spectral indices.

\begin{tabular}{lcccccc}
\hline \hline Src. & ID & $\begin{array}{c}\text { Right ascension } \\
(\mathrm{J} 2000)\end{array}$ & $\begin{array}{c}\text { Declination } \\
(\mathrm{J} 2000)\end{array}$ & $\begin{array}{c}1.4 \mathrm{GHz} \text { flux } \\
(\mathrm{mJy})\end{array}$ & $\begin{array}{c}322 \mathrm{MHz} \text { flux } \\
(\mathrm{mJy})\end{array}$ & $\begin{array}{c}\text { Spectral } \\
\text { index }\end{array}$ \\
\hline A & Northern Lobe & $23^{\mathrm{h}} 18^{\mathrm{m}} 06^{\mathrm{s}}$ & $+43^{\circ} 18^{\prime} 18^{\prime \prime}$ & $7.8 \pm 1.3$ & $20.0 \pm 2.0$ & $-0.6 \pm 0.3$ \\
B & Southern Lobe & $23^{\mathrm{h}} 18^{\mathrm{m}} 43^{\mathrm{s}}$ & $+43^{\circ} 12^{\prime} 20^{\prime \prime}$ & $5.5 \pm 0.5$ & $16.1 \pm 1.6$ & $-0.73 \pm 0.21$ \\
C & MCG+07-47-10 & $23^{\mathrm{h}} 18^{\mathrm{m}} 33^{\mathrm{s}}$ & $+43^{\circ} 14^{\prime} 49^{\prime \prime}$ & $3.9 \pm 0.4$ & $12.4 \pm 1.5$ & $-0.79 \pm 0.24$ \\
\hline
\end{tabular}

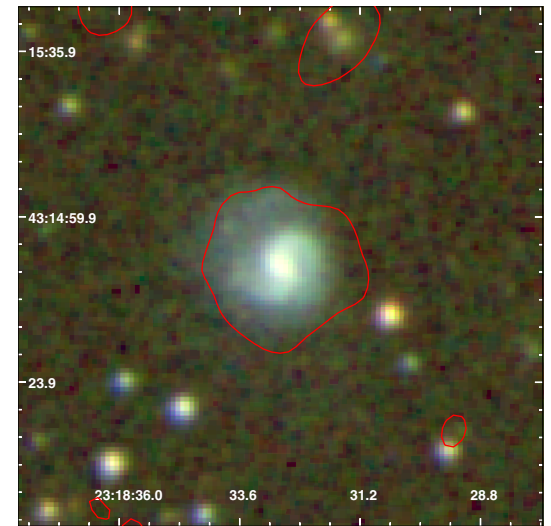

Fig. 3. Optical DSS image of the host galaxy (MCG+07-47-10) for the spiral DRAGN. The contour shows the $5 \sigma$ level of the $322 \mathrm{MHz}$ radio data.

redshift, MCG+07-47-10 would be located at a projected distance of $584 \mathrm{kpc}\left(28^{\prime}\right)$ from NGC 7618 and $375 \mathrm{kpc}\left(18^{\prime}\right)$ from UGC 12491. For a virial radius of these galaxy groups of approximately 600-800 kpc (Kraft et al. 2006), MCG+07-47-10 would therefore be found within the virial radius of both groups.

From the GMRT image, we find that this new spiral DRAGN has an angular linear extent of $\sim 9^{\prime}$, which equates to a physical size of $207 \mathrm{kpc}$ at our assumed redshift. Additionally, the optical diameter of MCG+07-47-10 is found to be $12.02 \mathrm{kpc}$.

At an assumed redshift of $z=0.017$ and with the measured radio flux density values in Table. 1 , we derive a radio luminosity at $322 \mathrm{MHz}$ for this proposed spiral DRAGN (lobes and host galaxy) of approximately $L_{322 \mathrm{MHz}}=3.15 \times 10^{22} \mathrm{Watts}^{-1}$ and $L_{1.4 \mathrm{GHz}}=1.12 \times 10^{22} \mathrm{Watts}^{-1}$. This is significantly weaker than other spiral DRAGNS such as $\mathrm{J} 1649+2635$ with $L_{1.4 \mathrm{GHz}}=$ $1.03 \times 10^{24}$ Watts $\mathrm{Hz}^{-1}$ (Mao et al. 2015).

MCG+07-47-10 and the two lobes are detected by the NVSS (Condon et al. 1998), but not by the VLA Low-frequency Sky Survey redux (VLSSr, Lane et al. 2014; Cohen et al. 2007),
TIFR GMRT Sky Survey (TGSS ADR, Intema et al. 2016), or the WEsterbork Northern Sky Survey (WENSS, Rengelink et al. 1997). In addition, the FIRST survey does not cover this area of the sky. In the NVSS, the three main components are detected, and the catalogue integrated fluxes for each component are shown in Table 1.

From the combination of the NVSS data and the GMRT data presented in this work, the host galaxy is found to have a spectral index of $\alpha=-0.79 \pm 0.24$, which is a typical value for a starforming spiral galaxies ( $\alpha=-0.74 \pm 0.03$, Gioia et al. 1982).

\section{Star formation rate}

MCG+07-47-10 is clearly detected in all four WISE bands (Wright et al. 2010). Based on colour-colour relationships (Lacy et al. 2004; Yan et al. 2013), the measured magnitude difference $[W 1]-[W 2]=0.184$ indicates that $\mathrm{MCG}+07-47-10$ is classified as a star-forming galaxy, with values greater than 0.8 signifying an AGN. Normal spiral galaxies are seen to have a range of magnitude differences of $\approx 0.0-0.6$ (Wright et al. 2010).

Using the derived relations between star formation rate (SFR) and observed luminosity in the WISE W3 and W4 bands (Jarrett et al. 2013), we find the SFR for MCG+07-47-10 at $12 \mu \mathrm{m}$ to be $\Sigma_{12}=0.75 M_{\odot} \mathrm{yr}^{-1}$ and at $22 \mu \mathrm{m}$ to be $\Sigma_{22}=$ $0.51 M_{\odot} \mathrm{yr}^{-1}$. In addition, MCG+07-47-10 is also detected in the near UV by GALEX (Martin et al. 2005). Following Kennicutt (1998), we find the UV SFR for MCG+07-47-10 to be $\Sigma_{\mathrm{UV}}=$ $0.16 M_{\odot} \mathrm{yr}^{-1}$. Assuming no contribution from an AGN, the SFR for MCG+07-47-10 derived from the radio data presented here is $\Sigma_{1.4 \mathrm{GHz}}=0.63 M_{\odot} \mathrm{yr}^{-1}$ (Condon 1992, Eq. (21)), intermediate to the IR and UV derived SFRs. There are large uncertainties associated with SFR indicators (Hopkins et al. 2003), therefore we estimate the SFR to be in the range of $0.16-0.75 M_{\odot} \mathrm{yr}^{-1}$.

The SFR rate in galaxies can show an enormous range from virtually zero in gas-poor ellipticals and dwarf-galaxies to $0.63 M_{\odot} \mathrm{yr}^{-1}$ in gas-rich spirals (Kennicutt 1998). For comparison, MCG+07-47-10 has an SFR similar to the nearby 
Sb galaxy, NGC2683, which has an $S F R \approx 0.36 M_{\odot} \mathrm{yr}^{-1}$ determined by Irwin et al. (2012). Compared to other spiral DRAGNS, Mao et al. (2015) found J1649+2635's SFR to be $\approx 0.26-2.6 M_{\odot} \mathrm{yr}^{-1}$. The two spiral DRAGNs found by Singh et al. (2015; J0836+0532,J1159+5820) were found to have 9.99 and $2.89 M_{\odot} \mathrm{yr}^{-1}$, respectively. $\mathrm{MCG}+07-47-10$ with an SFR range of $0.16-0.75 M_{\odot} \mathrm{yr}^{-1}$ would be in the lower range of the SFR of J1649+2635 and other spiral DRAGNs.

\section{Discussion and conclusions}

We presented the discovery of the spiral DRAGN MCG+07-4710 . The host galaxy has clearly defined spiral arms, a prominent bulge, and hosts a $188 \mathrm{kpc}$ DRAGN.

The radio source, first identified in NVSS, was not previously classified as a DRAGN, but rather as three separate radio sources. The deep GMRT observation detects the low surface brightness emission connecting the radio components, based on which we identify this radio source as a DRAGN for the first time.

The central component of the radio emission from the host galaxy appears extended in the NVSS and GMRT data. These new GMRT data show resolved radio emission emanating from the entirety of the host galaxy, as opposed to only the core. This suggests that the radio emission is not solely due to the presence of an AGN. Moreover, the total integrated radio flux density for the host galaxy gives an SFR that agrees well with SFRs calculated from the IR emission. Resolved radio emission observed throughout the disk suggests that most if not all the radio emission across the disk is due to star formation.

The luminosity of this spiral DRAGN $\left(L_{1.4 \mathrm{GHz}}=1.12 \times\right.$ $10^{22}$ Watts $\mathrm{Hz}^{-1}$ ) is significantly lower than that of other spiral DRAGNS. However, without a reliable redshift this could be misleading. Based on the median redshift from the main galaxy sample from SDSS of $z=0.1$ (Strauss et al. 2002), we would calculate a luminosity of $L_{1.4 \mathrm{GHz}}=4.25 \times 10^{23} \mathrm{Watts}^{\mathrm{Hz}}{ }^{-1}$. This is still lower than that found in other spiral DRAGNS such as $\mathrm{J} 1649+2635$ with $L_{1.4 \mathrm{GHz}}=1.03 \times 10^{24} \mathrm{Watts} \mathrm{Hz}^{-1}$ (Mao et al. 2015). A redshift of 0.1 would imply an optical diameter of $57 \mathrm{kpc}$ for MCG+07-47-10, and the angular linear extent of the entire spiral DRAGN of $\sim 9^{\prime}$ would equate to a physical size of $\approx 1 \mathrm{Mpc}$. For MCG+07-47-10 to have a luminosity similar to that of J1649+2635, MCG+07-47-10 would need to have a redshift of $z \approx 0.17$. This redshift would mean that the physical size of the DRAGN associated with MCG+07-47-10 is significantly larger than $1 \mathrm{Mpc}$. We note that this would not necessarily be unusual for the class, as two previously identified spiral DRAGNS (Hota et al. 2011; Bagchi et al. 2014) show evidence of megaparsec structure. However, the host galaxy, MCG+07-47-10, would then have an optical size of approximately $100 \mathrm{kpc}$, which would be exceptionally large compared to similar analogues.

The low-luminosity and low surface brightness of the DRAGN may suggest that the radio emission is old. One possible scenario leading to the formation of this spiral DRAGN is that the host of a low-luminosity DRAGN has had gas injected onto it, perhaps through a merger, and this gas has triggered star formation and built up spiral arms. Further modelling and observations at several frequencies are required to test this theory.

Assuming that the host galaxy, MCG+07-47-10, is associated with the two galaxy groups in its immediate vicinity (located within the virial radius of NGC 7618 and UGC 12491, Kraft et al. 2006), it would appear to reside in an intermediate density environment similar to other known spiral DRAGNs (Mao et al. 2015). This supports the idea that spiral DRAGNs require specific environments to form and that a moderately overdense environment is conducive for mergers to trigger a process that transitions ellipticals back to spirals.

In conclusion, we have presented the discovery of a new spiral DRAGN from observations with the GMRT at $322 \mathrm{MHz}$. We showed that the host galaxy is a star-forming spiral galaxy. Based on currently available information, we demonstrated that the radio luminosity of this new source is significantly lower than that of other spiral DRAGNs by about two orders of magnitude, with a value of $L_{1.4 \mathrm{GHz}}=1.12 \times 10^{22} \mathrm{Watts}_{\mathrm{Hz}}^{-1}$. This may indicate a previously unknown population of low-luminosity spiral DRAGNs, and we suggest that future radio surveys may be used to expand this sample further.

Acknowledgements. D.M., A.M.S. and A.C. gratefully acknowledge support from ERCStG 307215 (LODESTONE). M.Y.M. acknowledges support from EC H2020-MSCA-IF-2014 660432 (spiral DRAGNs). We thank the anonymous referee for their helpful comments that improved this paper. We acknowledge the usage of the HyperLeda database (http://leda.univ-lyon1.fr) and NASA/IPAC Extragalactic Database. The Digitized Sky Surveys were produced at the Space Telescope Science Institute under US Government grant NAG W-2166. The images of these surveys are based on photographic data obtained using the Oschin Schmidt Telescope on Palomar Mountain and the UK Schmidt Telescope. The plates were processed into the present compressed digital form with the permission of these institutions.

\section{References}

Alam, S., Albareti, F. D., Allende Prieto, C., et al. 2015, ApJS, 219, 12 Bagchi, J., Vivek, M., Vikram, V., et al. 2014, ApJ, 788, 174 Becker, R. H., White, R. L., \& Helfand, D. J. 1995, ApJ, 450, 559 Bennett, C. L., Larson, D., Weiland, J. L., \& Hinshaw, G. 2014, ApJ, 794, 135 Best, P. N., Kauffmann, G., Heckman, T. M., et al. 2005, MNRAS, 362, 25 Chiaberge, M., \& Marconi, A. 2011, MNRAS, 416, 917

Chiaberge, M., Gilli, R., Lotz, J. M., \& Norman, C. 2015, ApJ, 806, 147 Cohen, A. S., Lane, W. M., Cotton, W. D., et al. 2007, AJ, 134, 1245 Condon, J. J. 1992, ARA\&A, 30, 575

Condon, J. J., Cotton, W. D., Greisen, E. W., et al. 1998, AJ, 115, 1693 Gioia, I. M., Gregorini, L., \& Klein, U. 1982, A\&A, 116, 164 Hopkins, A. M., Miller, C. J., Nichol, R. C., et al. 2003, ApJ, 599, 971 Hopkins, P. F., Hernquist, L., Cox, T. J., \& Kereš, D. 2008, ApJS, 175, 356 Hota, A., Sirothia, S. K., Ohyama, Y., et al. 2011, MNRAS, 417, L36 Intema, H. T., van der Tol, S., Cotton, W. D., et al. 2009, A\&A, 501, 1185 Intema, H. T., Jagannathan, P., Mooley, K. P., \& Frail, D. A. 2016, A\&A, accepted [arXiv: 1603.04368]

Irwin, J., Beck, R., Benjamin, R. A., et al. 2012, AJ, 144, 43

Jarrett, T. H., Masci, F., Tsai, C. W., et al. 2013, AJ, 145, 6

Kennicutt, Jr., R. C. 1998, ARA\&A, 36, 189

Kraft, R. P., Jones, C., Nulsen, P. E. J., \& Hardcastle, M. J. 2006, ApJ, 640, 762 Lacy, M., Storrie-Lombardi, L. J., Sajina, A., et al. 2004, ApJS, 154, 166 Lane, W. M., Cotton, W. D., van Velzen, S., et al. 2014, MNRAS, 440, 327 Leahy, J. P. 1993, in Jets in Extragalactic Radio Sources, eds. H.-J. Röser, \& K. Meisenheimer (Berlin: Springer Verlag), Lect. Notes Phys. 421, 1 Ledlow, M. J., Owen, F. N., Yun, M. S., \& Hill, J. M. 2001, ApJ, 552, 120 Lintott, C. J., Schawinski, K., Slosar, A., et al. 2008, MNRAS, 389, 1179 Mao, M. Y., Owen, F., Duffin, R., et al. 2015, MNRAS, 446, 4176 Martin, D. C., Fanson, J., Schiminovich, D., et al. 2005, ApJ, 619, L1 Matthews, T. A., Morgan, W. W., \& Schmidt, M. 1964, ApJ, 140, 35 Meert, A., Vikram, V., \& Bernardi, M. 2015, MNRAS, 446, 3943

Rengelink, R. B., Tang, Y., de Bruyn, A. G., et al. 1997, A\&AS, 124 Scaife, A. M. M., \& Heald, G. H. 2012, MNRAS, 423, L30 Singh, V., Ishwara-Chandra, C. H., Sievers, J., et al. 2015, MNRAS, 454, 1556 Strauss, M. A., Weinberg, D. H., Lupton, R. H., et al. 2002, AJ, 124, 1810 Swarup, G. 1990, Indian J. Radio Space Phys., 19, 493 Urry, C. M., \& Padovani, P. 1995, PASP, 107, 803

Vorontsov-Vel'Yaminov, B. A., \& Arkhipova, V. P. 1968, Trudy Gosudarstvennogo Astronomicheskogo Instituta, 38, 1 Wright, E. L., Eisenhardt, P. R. M., Mainzer, A. K., et al. 2010, AJ, 140, 1868 Yan, L., Donoso, E., Tsai, C.-W., et al. 2013, AJ, 145, 55 